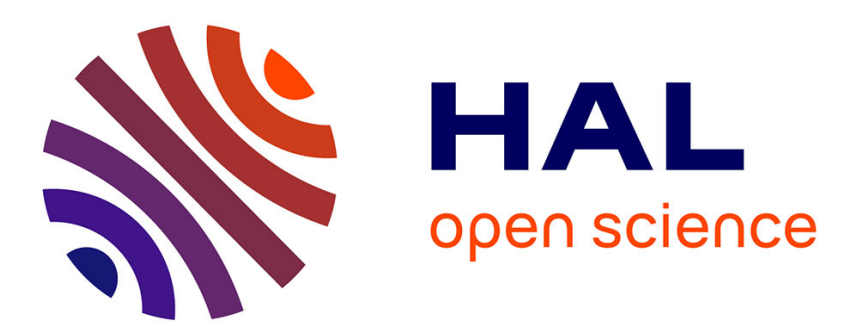

\title{
Robust Optimal Scheduling of Combined Chemo-and Immunotherapy: Considerations on Chemotherapy Detrimental Effects
}

Kaouther Moussa, Mirko Fiacchini, Mazen Alamir

\section{- To cite this version:}

Kaouther Moussa, Mirko Fiacchini, Mazen Alamir. Robust Optimal Scheduling of Combined Chemoand Immunotherapy: Considerations on Chemotherapy Detrimental Effects. ACC 2020 - American Control Conference, Jul 2020, Denver (online), United States. 10.23919/ACC45564.2020.9147869 . hal-02871352

\section{HAL Id: hal-02871352 \\ https://hal.science/hal-02871352}

Submitted on 17 Jun 2020

HAL is a multi-disciplinary open access archive for the deposit and dissemination of scientific research documents, whether they are published or not. The documents may come from teaching and research institutions in France or abroad, or from public or private research centers.
L'archive ouverte pluridisciplinaire HAL, est destinée au dépôt et à la diffusion de documents scientifiques de niveau recherche, publiés ou non, émanant des établissements d'enseignement et de recherche français ou étrangers, des laboratoires publics ou privés. 


\title{
Robust Optimal Scheduling of Combined Chemo- and Immunotherapy: Considerations on Chemotherapy Detrimental Effects
}

\author{
Kaouther Moussa*, Mirko Fiacchini* and Mazen Alamir*
}

\begin{abstract}
In this paper, we investigate a mathematical model describing interactions between cancer and the immune system. In this model, we take into account the detrimental effects of chemotherapy on both populations (cancer and immune cells) and incorporate the beneficial effects of the immune system in controlling the tumor growth. The problem of cancer treatment scheduling is considered as a robust optimal control problem (ROCP) in the sense that we derive statistically optimal combined strategies of chemo- and immunotherapy treatments, assuming the knowledge of the probability distribution of the chemotherapy killing parameter (effects on the immune population). Furthermore, we add in the ROCP a health constraint on the minimal allowed immune cells density. We use the moments optimization framework, which allows to consider uncertainties on model parameters implicitly.
\end{abstract}

\section{INTRODUCTION}

In the last decades, researchers had been interested in modeling the interaction dynamics between cancer and the human body in order to better understand and to analyse the behavior of these phenomena. Since the dynamics of cancer growth are extremely complex, we can find many different models in the literature, depending on the therapies that are used, for example, or the different phenomena that occur in the human body. According to [5], for the specific case of cancer-immune interactions, the mathematical modeling of the entire immune system can be a very complex task, that is one of the reasons that researchers focus on the elements of the immune system that are known to be significant in controlling the tumor growth.

Several works had been done on modeling the interaction of chemotherapy with the tumor growth process, for instance, [16], [20] and [1]. Moreover, there are some models considering specific phenomena, for instance [4], where authors took into account the influence of nutrients on the drug effect or [10], where authors considered the common phenomenon of cancer cells resistance to chemotherapy. Furthermore, with the development of new cancer therapies such as immunotherapy and antiangiogenic therapy, other recent models describing the interaction of these drugs with the tumor growth have been developed. In particular, the recent advances in

* Univ. Grenoble Alpes, CNRS, Grenoble INP, GIPSA-lab, 38000 Grenoble, France \{kaouther.moussa,mirko.fiacchini, mazen.alamir\}@gipsa-lab.fr genetics led to considerable progress in experimental and clinical immunology [9] and many researches on modeling the immune system dynamics had been carried out. The readers interested in tumor-immune interactions modeling can refer to [6], [8], [9] and references therein.

This progress in cancer dynamics modeling motivated researchers to apply control approaches in order to schedule cancer treatments using sometimes optimal control strategies. We can find in the literature many works regarding the application of optimal control approaches on cancer treatment problems. For instance, [21] and [7], where optimal protocols for anti-angiogenic therapy were investigated, or [6] where quadratic and linear controls were designed for a tumor-immune interactions model with chemotherapy delivery. However, only few works addressed the problem of handling parametric uncertainties. We cite for example, [2] where a robust feedback scheme is proposed to schedule antiangiogenic treatment combined with chemotherapy, [12] where an $H_{\infty}$ based robust control was applied to the same model and [3] where a general framework for probabilistic certification of cancer therapies was proposed.

As an extension of our work in [19], where we derived robust optimal control strategies for combined chemoand immunotherapy treatments, considering uncertainties description on the tumor growth rate and the rate of immune cells influx, in this paper, we investigate the consequences, in the drug scheduling, of adding a new term standing for the effect of chemotherapy on immune cells. Furthermore, we consider that this parameter is uncertain and described by a probability distribution. We propose to formulate robust optimal control problems in the moment optimization framework. Since the problem is reformulated in the space of probability measures, it is straightforward to define states and parameters as random variables characterized by their probability distributions. The resulting infinite-dimensional problem is solved using truncations known as finite-dimensional semidefinite (SD) hierarchies [13], providing a converging sequence of lower bounds on the optimal solution.

In Section II, we present the dynamical model that we used for numerical simulations. Section III recalls the basic concepts of the generalized moment problem for optimal control. The optimal control problems to be solved and simulation results are presented in Section IV. Finally, in Section V, we discuss the main advantages 


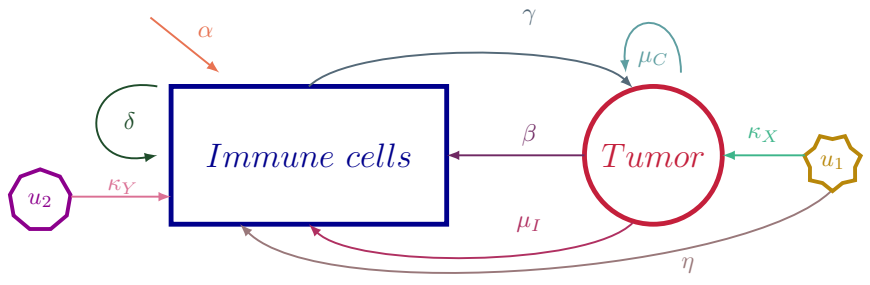

Fig. 1: A scheme showing the interactions in model (1), between the tumor and the immune system

and limitations of this approach and we present some perspectives for future works.

\section{DyNAMiCAL MODEL}

According to [15], mathematical models for tumor immune interactions have a long history dating back to Stepanova's model [23]. The latter gives the advantage of a minimally parametrized model that nevertheless includes the main aspects of cancer-immune interactions. The model presented in [23] had been intensively used in the literature with different growth functions, in order to investigate its equilibria and propose some optimal control strategies. However, the effect of the cytotoxic agent on the immuno-competent cell's density was neglected. We cite for instance [22], where authors proposed a multiple model predictive control scheme for a model with gompertzian tumor growth rate.

Let's consider the following dynamics:

$$
\begin{aligned}
& \dot{x}_{1}=\mu_{C} x_{1}-\frac{\mu_{C}}{x_{\infty}} x_{1}^{2}-\gamma x_{1} x_{2}-\kappa_{X} x_{1} u_{1}, \\
& \dot{x}_{2}=\mu_{I} x_{1} x_{2}-\beta x_{1}^{2} x_{2}-\delta x_{2}+\kappa_{Y} x_{2} u_{2}-\eta u_{1} x_{2}+\alpha,
\end{aligned}
$$

where $x_{1}$ and $x_{2}$ denote, respectively, the number of tumor cells and the density of effector immune cells (ECs), $u_{1}$ and $u_{2}$ are, respectively, the delivery profiles of a cytotoxic agent and an immunostimulator. Fig. 1 presents a scheme describing the different interactions between the tumor and the immune system.

Similarly to the model that we investigated in [19], we consider in this paper a modified version of Stepanova's model [23], where we replace the exponential growth term by a logistic one $\left(f\left(x_{1}\right)=\mu_{C} x_{1}\left(1-\frac{x_{1}}{x_{\infty}}\right)\right)$, since the logistic term allows to consider a limited tumor volume which is more realistic than the exponential growth. Unlike the model considered in [19], we added in model (1) the term $-\eta u_{1} x_{2}$ in the dynamics $\dot{x}_{2}$, which stands for the direct detrimental effects that chemotherapy has on the immune system. Table I summarizes the definitions of the other model parameters and their numerical values.

As shown in Fig. 2, the uncontrolled model (1) has two locally asymptotically stable equilibria. The macroscopic malignant equilibrium is $\left(x_{m}, y_{m}\right)=(735.9,0.032)$ and the benign one is $\left(x_{b}, y_{b}\right)=(34.98,0.53)$. The trajectory
TABLE I: Numerical values and definitions of the pa-

\begin{tabular}{|c|c|c|}
\hline Parameter & Definition & Numerical value \\
\hline$\mu_{C}$ & tumor growth rate & $0.5599 \cdot 10^{7}$ cells/day \\
\hline$\mu_{I}$ & $\begin{array}{l}\text { tumor stimulated } \\
\text { proliferation rate }\end{array}$ & 0.00484 day $^{-1}$ \\
\hline$\alpha$ & $\begin{array}{l}\text { rate of immune } \\
\text { cells influx }\end{array}$ & $0.1181 \mathrm{day}^{-1}$ \\
\hline$\beta$ & inverse threshold & 0.00264 \\
\hline$\gamma$ & interaction rate & $1 \cdot 10^{7}$ cells/day \\
\hline$\delta$ & death rate & $0.37451 \mathrm{day}^{-1}$ \\
\hline$\kappa_{X}$ & $\begin{array}{l}\text { chemotherapeutic } \\
\text { killing parameter }\end{array}$ & $1 \cdot 10^{7}$ cells/day \\
\hline$\kappa_{Y}$ & $\begin{array}{l}\text { immunotherapy } \\
\text { injection parameter }\end{array}$ & $1 \cdot 10^{7}$ cells/day \\
\hline$x_{\infty}$ & fixed carrying capacity & $780 \cdot 10^{6}$ cells \\
\hline
\end{tabular}
rameters used in model (1) [8]

in black represents the evolution of uncontrolled states starting from the initial condition $x_{0}=(500,0.5)$.

It is important to notice that the treatment performance depends highly on the initial conditions, since there is coexistence of macro- and microscopic equilibria. The initial states of system (1) can be estimated with some uncertainties, before designing the drug injection schedules. The objective of cancer treatment can be formulated as to drive the state initial conditions from the region of attraction of the malignant equilibrium to the region of attraction of the benign equilibrium.

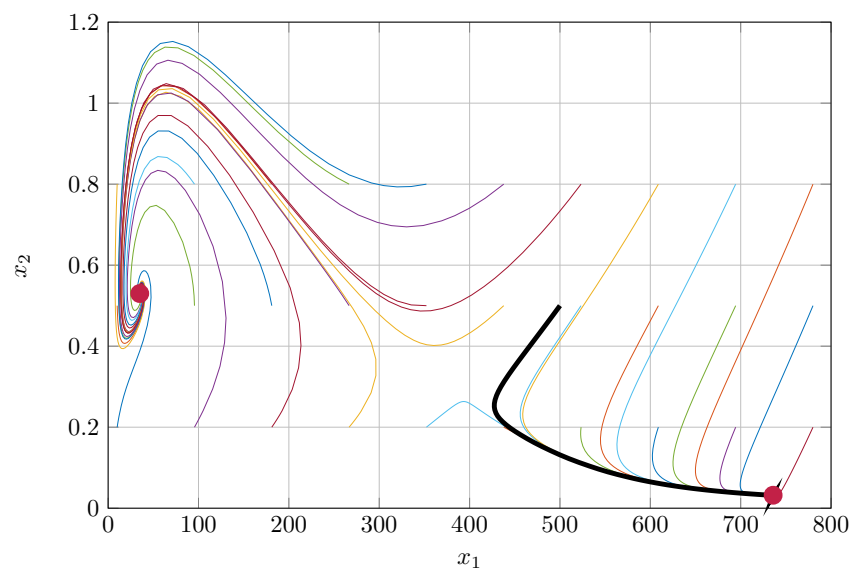

Fig. 2: Phase portrait of model (1).

\section{OvERVIEW ON GENERALIZED MOMENT PROBLEM FOR OPTIMAL CONTROL}

In this section we will present an overview on the main key points of the generalized moment problem for solving optimal control problems. This approach, developed by Lasserre [13], is based on the fact that polynomial optimization problems (which are a class of nonconvex finite dimensional problems) are equivalent, in the space of measure, to infinite dimensional problems, under mild assumptions. Those infinite dimensional problems are nevertheless linear and can be solved using 
relaxations (known as Lasserre hierarchy [13]) which provide a converging sequence of lower bounds on the global optima, under some compactness assumptions. Therefore, generating and solving these relaxations allow to approach the exact solution of the original polynomial optimization problem with arbitrary precision.

This approach has been extended recently to optimal control problems with a polynomial structure and bounded constraints [14], for which suboptimal solutions, converging to the exact optimal control, can be obtained by solving sequences of convex problems. Moreover, as the linear infinite dimensional problems are defined in the space of measures, this approach allows to address optimal control problems which involve probability distributions as states and parameters.

Consider the following polynomial optimal control problem:

$$
\begin{array}{ll}
\inf _{u(\cdot)} & \int_{0}^{T} L(t, x(t), u(t)) d t+\Phi(x(T)) \\
\text { s.t. } & \dot{x}(t)=f(t, x(t), u(t)) \\
& x(t) \in X, u(t) \in U, t \in[0, T] \\
& x(0) \in X_{0}, x(T) \in X_{T}
\end{array}
$$

where $x \in \mathbb{R}^{n}$ is the state, $u \in \mathbb{R}^{m}$ is the input, functions $f: \mathbb{R} \times \mathbb{R}^{n} \times \mathbb{R}^{m} \rightarrow \mathbb{R}, L: \mathbb{R} \times \mathbb{R}^{n} \times \mathbb{R}^{m} \rightarrow \mathbb{R}$ and $\Phi: \mathbb{R}^{n} \rightarrow \mathbb{R}$ are polynomials and the constraints sets $X_{0}, X, X_{T}$ and $U$ are compact basic semi-algebraic sets, i.e. defined as the union of finitely many intersections of closed polynomial superlevel sets. The optimum of (2) is the same as the optimum of a linear infinite dimensional optimization problem defined over the space of probability measures (given below), under an additional assumption on the convexity of the set $f(t, x, U)$ for all $t$ and $x$.

For this, we introduce briefly the definitions of measure and moments, the reader interested in more details regarding this topic is referred to the related literature, e.g. [13] and [14].

Given a compact set $X \in \mathbb{R}^{n}$ and the Borel $\sigma$ algebra $\mathcal{B}(X)$, which is a particular set of subsets of $X$ containing all the open subsets of $X$, a Borel measure $\mu$ on $X$ is a function that associates a real value to every element of $\mathcal{B}(X)$. A measure is nonnegative if it takes only nonnegative values, is a probability measure if it is nonnegative and $\mu(X)=1$. An example of a positive measure is the Dirac measure $\delta_{w}(X)$ with $w \in X$ which assigns the value 1 to every subset of $X$ which contains $w$ and 0 otherwise. The spaces of measures and positive measures are denoted $\mathcal{M}(X)$ and $\mathcal{M}_{+}(X)$, respectively. Measures can also be defined as the space of linear functionals acting on the space of functions which are continuous on $X$, i.e. by the action they have over the elements of the dual space $\mathcal{C}(X)$ through integration

$$
\langle v, \mu\rangle=\int_{X} v(x) \mu(d x)
$$

for all $v \in \mathcal{C}(X)$. The following linear problem in the space of measures

$$
\begin{array}{lc}
\inf _{\mu_{0}, \mu, \mu_{T}} & \langle L, \mu\rangle+\left\langle\Phi, \mu_{T}\right\rangle \\
\text { s.t. } & \int_{[0, T] \times X \times U}\left(\frac{\partial v(t, x)}{\partial t}+\nabla_{x}(v(t, x))^{\prime} f(t, x, u)\right) d \mu \\
& =\left\langle v, \mu_{T}\right\rangle-\left\langle v, \mu_{0}\right\rangle, \quad \forall v \in \mathcal{C}^{1}([0, T] \times X) \\
& \mu_{0} \in \mathcal{M}_{+}\left(\{0\} \times X_{0}\right), \mu_{T} \in \mathcal{M}_{+}\left(\{T\} \times X_{T}\right) \\
& \mu \in \mathcal{M}_{+}([0, T] \times X \times U), \\
& \left\langle 1, \mu_{0}\right\rangle=1,
\end{array}
$$

is infinite dimensional and has the same optimum value as the original optimal control problem (2), under mild assumptions, see [14]. This problem remains highly complex, however, Lasserre hierarchy [13] of relaxed LMI problems can be determined to obtain suboptimal solutions that converge to the optimal solution of the original optimal control problem, under some compactness and convexity assumptions. In order to obtain the relaxations, one has first to consider the relation between the measure $\mu_{0}, \mu$ and $\mu_{T}$ and their moments. Given $x \in \mathbb{R}^{n}$ and $\sigma \in \mathbb{N}^{n}$, the moment of order $\sigma \in \mathbb{N}^{n}$ of $\mu \in \mathcal{M}(X)$ is defined as:

$$
y_{\sigma}=\int_{X} x^{\sigma} \mu(d x)=\left\langle x^{\sigma}, \mu\right\rangle,
$$

where $x^{\sigma}=\prod_{k=1}^{n} x_{k}^{\sigma_{k}}$. LMI conditions can be given in terms of the moments of $\mu$ that are equivalent to the constraint $\mu \in \mathcal{M}_{+}(X)$, conditions that still involve infinite dimensional matrices which are functions of the infinitely many variables $y_{\sigma}$ for all $\sigma \in \mathbb{N}^{n}$. The relaxations consist in considering the matrix structures obtained by appropriately truncating the vector of moments to a finite maximal degree $(r)$ and imposing in (3) constraints over polynomials of a finite maximal degree in spite of all $v \in \mathcal{C}^{1}([0, T] \times X)$. This leads to a hierarchy of finitedimensional semidefinite programming problems whose solutions converge to the solution of the optimal control problem as the relaxation degree grows.

The interesting feature of this approach is the fact that, even in case of deterministic dynamical systems, the initial state as well as the final one and the state along trajectories, are dealt with by defining measures on the state space, see (3). The same holds for the input. For instance, if $x_{0}=x(0) \in X_{0}$ is a singleton, then the initial measure $\mu_{0}$ in (3) should be imposed by fixing, for all $\sigma \in \mathbb{N}^{n}$, its moments given as:

$$
\left\langle t^{\iota} x^{\sigma}, \mu_{0}\right\rangle= \begin{cases}x_{0}^{\sigma} & \text { if } \iota=0 \\ 0 & \text { if } \iota \in \mathbb{N}^{+} \backslash\{0\}\end{cases}
$$

Therefore, this method is suitable for dealing with states and inputs that are characterized by probability distributions, simply by imposing the moments of the related probability distribution functions.

In the particular case under study, we want to design a robust optimal control for a dynamical model describing 
interaction between cancer and the immune system, the parameter which is supposed to be not perfectly known is the chemotherapy-induced damage on immune cells. This lack of knowledge can be modeled through an uncertain parameter characterized by a probability distribution, with compact support. Then, in practice, it is sufficient to define an extended state containing tumor and immune cell populations in addition to the uncertain parameter, i.e. $\eta$, see (1), and to impose its time invariant characteristic through the dynamics $\dot{\eta}(t)=0$. Thus, supposing that $\nu(\eta)$ denote the probability distribution of $\eta$, the optimal control problem (3) to be solved should have as initial condition $\mu_{0}\left(t, x_{1}, x_{2}, \eta\right)=\delta_{0}(t) \times \delta_{x_{1}(0)}\left(x_{1}\right) \times$ $\delta_{x_{2}(0)}\left(x_{2}\right) \times \nu(\eta)$, imposed through moments of the initial measure.

\section{ROCP FOR CANCER THERAPY DESIGN}

We will first consider a nominal value of $\eta$ and solve the optimal control problem, then we will define $\eta$ as an uncertain parameter, with a given probability distribution, and solve the robust optimal control problem. Finally, we compare the effect of both profiles (nominal and robust) on dynamics, in order to infer on the consequences of adding the chemotherapy-induced damage term and to highlight the importance of considering it in the therapy scheduling design.

Let's consider that the initial condition is $\left(x_{10}, x_{20}\right)=$ $(500,0.5)$, we can see in Fig. 2 that without control, the trajectory corresponding to this initial state converges to the malignant equilibrium.

\section{A. Nominal optimal control problem}

We consider that the maximum drug dose is 1 for both chemotherapy and immunotherapy. Furthermore, we add constraints on the immune cells density and the number of tumor cells in order to ensure the compactness of the state set $X$. We also impose a constraint on the final tumor size in order to drive the tumor to the benign region and a constraint on the minimal immune cells density, recommended to prevent the body from any weakening of the immune system. The nominal (i.e. considering a nominal value of $\eta$ ) optimal control problem that we propose to solve for $t \in[0,60]$ (days) is the following:

$$
\begin{array}{ll}
\min _{u_{1}(\cdot), u_{2}(\cdot)} & J\left(x_{1}, x_{2}, u_{1}, u_{2}\right) \\
\text { s.t. } & \dot{x}_{1}=\mu_{C} x_{1}\left(1-\frac{x_{1}}{x_{\infty}}\right)-\gamma x_{1} x_{2}-\kappa_{X} x_{1} u_{1}, \\
& \dot{x}_{2}=\mu_{I}\left(x_{1}-\beta x_{1}^{2}\right) x_{2}-\delta x_{2}+\alpha+\kappa_{Y} x_{2} u_{2} \\
& \quad-\eta u_{1} x_{2}, \\
& x_{1}(0)=500, x_{2}(0)=0.5 \\
& x_{1}(60) \leqslant 100, \\
& 0 \leqslant u_{1} \leqslant 1,0 \leqslant u_{2} \leqslant 1, \\
& 0 \leqslant x_{1} \leqslant 780,0 \leqslant x_{2} \leqslant 5 \\
& x_{2} \geqslant 0.1 \\
& t \in[0,60] .
\end{array}
$$

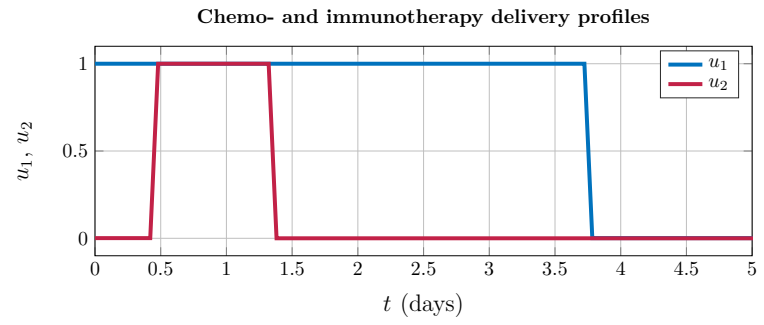

Fig. 3: Nominal control inputs $\left(u_{1}\right.$ and $\left.u_{2}\right)$, for $\eta=1$.

The cost $J$ is chosen according to the objectives that one seeks to achieve. It can contain many terms such as final states, integrals of state trajectories and control inputs, with different penalties in order to achieve a trade-off between the different control objectives. Problem (5) has been reformulated in the framework of moments optimization via GloptiPoly 3 [11], as explained in Section III, and has been solved using YALMIP and the semidefinite programming solver MOSEK. The control inputs are approximated, based on the knowledge of their moments, using Christoffel-Darboux kernel, for more details, see [17]. For practical reasons, time and states trajectories are scaled to $[0,1]$, therefore, the control inputs presented in this paper are computed for scaled dynamics.

Since chemotherapy has damaging side effects on the human body, it is common to frame an optimal control problem so that the total amount of drugs is minimized [6]. It is also important to minimize the use of immunotherapy since the available amount is limited, and for some treatment types, immunotherapy can even be toxic [18]. Thereby, one can easily notice that the choice of the cost $J$, to be minimized, is very important in order to meet the control objectives. Here, we focus on the assessment of the methodology by taking the following cost:

$J=10 x_{1}(60)+4 \int x_{1}(t) d t+0.01 \int u_{1}(t) d t+0.1 \int u_{2}(t) d t$.

In Fig. 3, we show the drug delivery profiles in the time interval $[0,5]$ to highlight the treatment duration, since for $t \in[5,60], u_{1}(t)=0$ and $u_{2}(t)=0$. We can see in this figure that, for $\eta=1$, the chemotherapy profile is a considerable injection at the beginning of treatment followed by a one day maximal dose injection of immunotherapy.

Fig. 4 shows the time evolution of state trajectories, we can notice that the tumor burden is considerably reduced during the 5 first days, this is due to the considerable amount of chemotherapy drugs injected at the beginning. We can also notice that this important injection of chemotherapy induced a decrease in the density of immune cells (due to the term $-\eta u_{1} x_{2}$ ) in the dynamics of $x_{2}$. However, the minimal constraint is still respected thanks to the immunostimulation $\left(u_{2}\right)$.

Even though controls in Fig. 3 satisfy standard objectives in the context of nominal optimal control, we will 

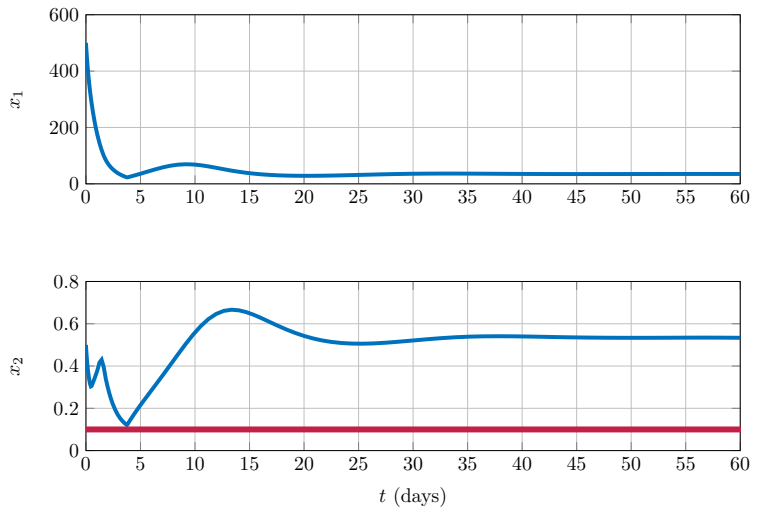

Fig. 4: States trajectories with nominal control profiles

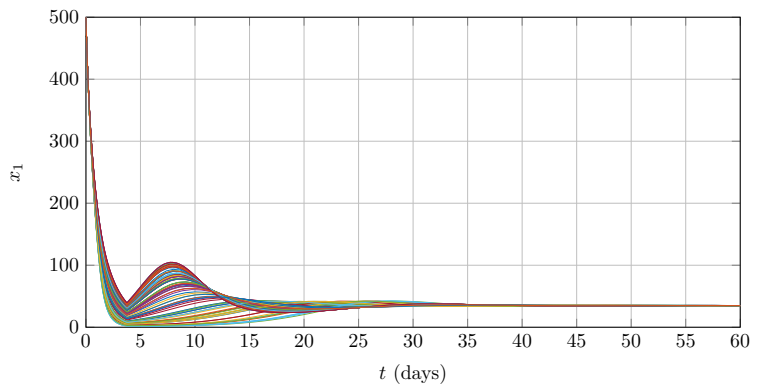

Fig. 5: MC tests on nominal profiles, $x_{1}$ trajectory

show that when the dynamics are subject to parametric uncertainties, those controls will not meet the goals set in the optimal control problem.

Let's assume that $\eta \sim \mathcal{U}([0,2])$.

Remark 1: The considered distribution is not based on practical knowledge of the system parameters, it is chosen only to illustrate the problem of handling parametric uncertainties. Of course, the method can be applied to general probability distributions.

Fig. 5 and Fig.6 presents 100 Monte-Carlo (MC) simulations with random values of $\eta$. It shows that there are many violations of immune cells density constraint (i.e. leading to critical immune weakening of patients). Another point to notice is that, in some cases, there is a small tumor regrowth due to the weakening of immune system. Therefore, it is crucial to consider the potential uncertainties on model parameters.

\section{B. Robust optimal control problem}

Let's extend system (1) to the following dynamics:

$$
\begin{aligned}
\dot{x}_{1} & =\mu_{C} x_{1}-\frac{\mu_{C}}{x_{\infty}} x_{1}^{2}-\gamma x_{1} x_{2}-\kappa_{X} x_{1} u_{1}, \\
\dot{x}_{2} & =\mu_{I}\left(x_{1}-\beta x_{1}^{2}\right) x_{2}-\delta x_{2}+\kappa_{Y} x_{2} u_{2}+\alpha-\eta u_{1} x_{2}, \\
\dot{\eta} & =0 .
\end{aligned}
$$

The state extension in (6) allows to characterize $\eta$ by its probability distributions, as explained in Section III. Similarly to problem (5), one can reformulate the robust

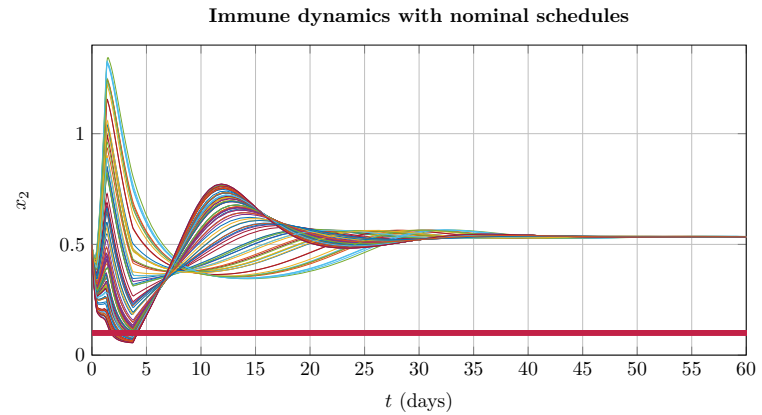

Fig. 6: MC tests on nominal profiles, $x_{2}$ trajectory

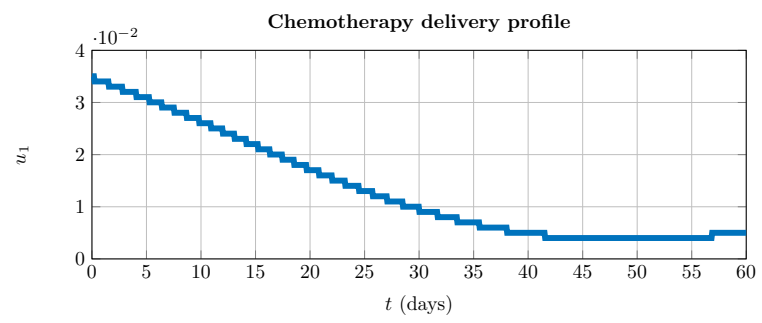

Fig. 7: Robust chemotherapy control input $\left(u_{1}\right)$

optimal control problem with dynamics (6) by including the moments of the distribution of $\eta$.

Fig. 7 shows the robust chemotherapy injection profile, we can notice that compared to the nominal case (Fig. 3), the use of chemotherapy in the robust case has been considerably reduced in intensity but extended in time. This is due to the presence of constraint on the minimal immune cells density and the new term $-\eta u_{1} x_{2}$ which reduces the amount of immune cells when chemotherapy concentration increases. The robust chemotherapy schedule uses less than $3.5 \%$ of the maximal allowed dose, this concentration decreases slowly during the treatment period while in the nominal profile, it is a one maximal dose at the beginning of treatment period.

Remark 2: We can notice that there is continuous administration of chemotherapy in both profiles (nominal and robust) which is unrealistic in practice. However, this methodology remains interesting since it provides a guide on the maximal concentrations to be injected and their evolution with respect to time. Furthermore, these profiles can be approximated using impulses in order to be applicable from a practical point of view.

Fig. 8 shows the immunotherapy profile, we can notice that the nominal and robust profiles of immunotherapy are almost the same, it is a one day maximal dose at the beginning of the treatment period.

Similarly to the nominal case, we did 100 Monte-Carlo simulations on system (1), using robust schedules, results are presented in Fig. 9 and 10. We can notice in Fig. 9 that the tumor volume takes more time to be reduced in the robust case. However, as we can see in Fig. 10, there is no immune constraints violation unlike the nominal case. 


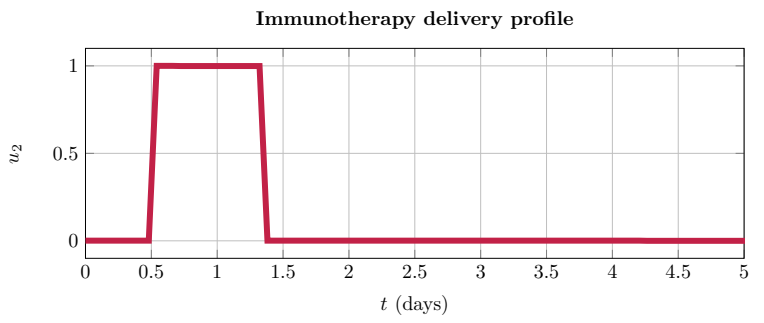

Fig. 8: Robust immunotherapy control input $\left(u_{2}\right)$

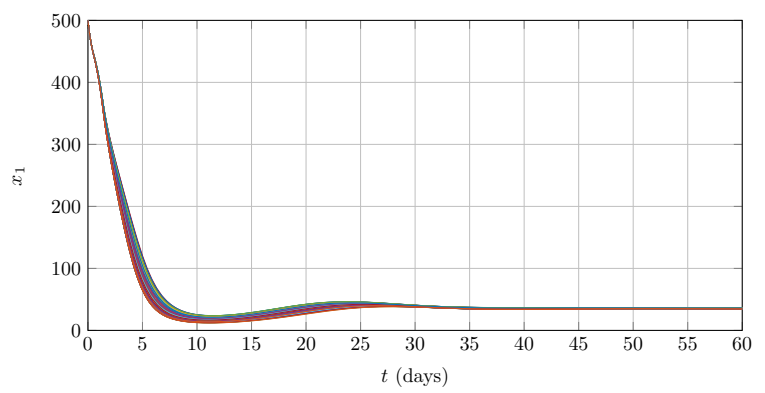

Fig. 9: MC tests on robust profiles, $x_{1}$ trajectory

\section{Conclusion}

We presented in this paper some results on the application of moment optimization theory to schedule cancer treatment. We highlighted the importance of taking into account the side effects of chemotherapy on immune cells, we notice that when we consider those effects in addition to a constraint on the minimal allowed immune cells density, the concentration of the injected cytotoxic agent is considerably reduced.

The moment optimization approach is promising for many applications, since it allows to solve optimal control problems for a class of nonlinear systems, with uncertainties on parameters and initial states. However, it may have some limitations, mainly the restriction on polynomial dynamics and the limited dimension (state and control variables) that can be handled. Finally, future work will be focused on considering uncertainties on state initial conditions and applying this methodology to other models of cancer dynamics.

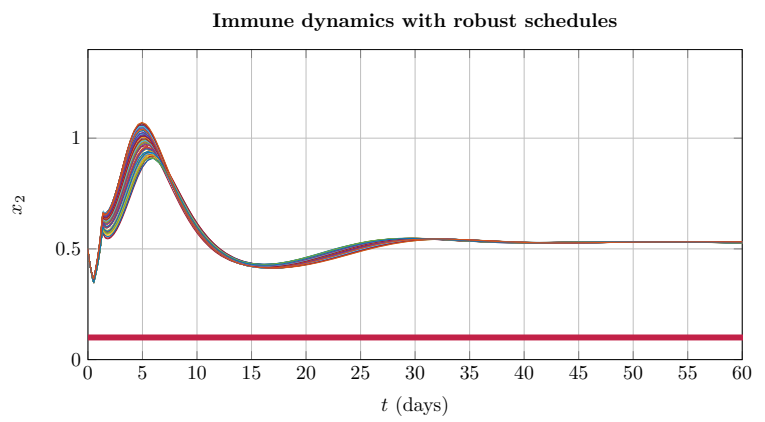

Fig. 10: MC tests on robust profiles, $x_{2}$ trajectory

\section{REFERENCES}

[1] E. Afenya. Acute leukemia and chemotherapy: A modeling viewpoint. Mathematical Biosciences, 138(2):79-100, 1996.

[2] M. Alamir. Optimal Control Applications And Methods Robust Feedback Design For Combined Therapy of Cancer. Optim. Control Appl. Meth, 2014.

[3] M. Alamir. On Probabilistic Certification of Combined Cancer Therapies Using Strongly Uncertain Models. Journal of Theoretical Biology, Elsevier, 384:59-69, 2015.

[4] A. Bratus, I. Samokhin, I. Yegorov, and D. Yurchenko. Maximization of viability time in a mathematical model of cancer therapy. Mathematical Biosciences 294, 2017.

[5] L. G. De Pillis. Mixed Immunotherapy and Chemotherapy of Tumors : Modeling, Applications and Biological Interpretations . Journal of Theoretical Biology, 2006.

[6] L. G. De Pillis, W. Gu, K. R. Fister, T. Head, K. Maples, A. Murugan, T. Neal, and K. Yoshida. Chemotherapy for tumors: An analysis of the dynamics and a study of quadratic and linear optimal controls. Mathematical Biosciences, 2007.

[7] A. d'Onofrio and P. Cerrai. A Bi-parametric Model for The Tumour Angiogenesis and Antiangiogenesis Therapy. Mathematical and Computer Modelling, 2009.

[8] A. d'Onofrio, U. Ledzewicz, and H. Schättler. On the Dynamics of Tumor-Immune System Interactions and Combined Chemo- and Immunotherapy. Springer Milan, 2012.

[9] R. Eftimie, J. J. Gillard, and D. A. Cantrell. Mathematical Models for Immunology: Current State of the Art and Future Research Directions. Bulletin of Mathematical Biology, 2016.

[10] M. Feizabadi. Modeling multi-mutation and drug resistance: analysis of some case studies. Theoretical Biology and Medical Modelling, 2017.

[11] D. Henrion, J. B. Lasserre, and J. Löfberg. Gloptipoly 3: Moments, optimization and semidefinite programming. Optim. Methods and Software, 2009.

[12] L. Kovacs, A. Szeles, J. Sapi, D. A. Drexler, I. Rudas, I. Harmati, and Z. Sapi. Model-based angiogenic inhibition of tumor growth using modern robust control method. Computer Methods and Programs in Biomedicine, 2014.

[13] J. B. Lasserre. Moments, positive polynomials and their applications. Imperial College Press, London, UK, 2010.

[14] J. B. Lasserre, D. Henrion, C. Prieur, and E. Trélat. Nonlinear optimal control via occupation measures and LMI relaxations. SIAM J. Control Opt., 2008.

[15] U. Ledzewicz and M. Faraji. On Optimal Protocols for Combinations of Chemo- and Immunotherapy. In 51st IEEE Conference on Decision and Control, Hawaii, USA, December 10-13 2015 .

[16] R. Martin. Optimal control drug scheduling of cancer chemotherapy. Automatica, 28(6):1113-1123, 1992.

[17] S. Marx, E. Pauwels, T. Weisser, D. Henrion, and J. Lasserre. Tractable semi-algebraic approximation using Christoffel-Darboux kernel. http://homepages.laas.fr/henrion/papers/momgraph.pdf, 2019.

[18] J. Mier, F. Aronson, R. Numerof, G. Vachino, and M. Atkins. Toxicity of immunotherapy with interleukin-2 and lymphokine-activated killer cells. Pathology and Immunopathology Research, 1988.

[19] K. Moussa, M. Fiacchini, and M. Alamir. Robust Optimal Control-based Design of Combined Chemo- and Immunotherapy Delivery Profiles. In 8th IFAC Conference on Foundations of Systems Biology in Engineering, València, Spain, October 15-18 2019.

[20] J. M. Murray. Optimal control cancer growth. Mathematical Biosciences, 98:273-287, 1990.

[21] H. Schättler, U. Ledzewicz, and B. Cardwell. Robustness of Optimal Controls For a Class of Mathematical Models for Tumor Anti-Angiogenesis. 2011.

[22] N. Sharifi, S. Ozgoli, and A. Ramezani. Multiple model predictive control for optimal drug administration of mixed immunotherapy and chemotherapy of tumours. Computer Methods and Programs in Biomedicine, 2017.

[23] N. Stepanova. Course of the immune reaction during the development of a malignant tumour. Biophysics, 1980. 